\title{
Étude de la prévision de la digestibilité des rations mixtes distribuées à des chèvres laitières par ses caractéristiques analytiques
}

\author{
Sylvie GIGER, D. SAUVANT, J. HERVIEU ct Michelle DORLEANS \\ INRA, Station de Nutrition et Alimentation \\ I.N.A. Paris-Grignon, 16, rue Claude-Bernard, F 75231 Paris Cedex 05
}

\begin{abstract}
Résumé
L'objet de ce travail est la recherche des principaux facteurs de variation de la digestibilité de la matière organique des rations mixtes distribuées à des chèvres laitières, et les moyens de les prévoir. Il a été effectué à partir de la banque de données expérimentales du laboratoire INRA de la Chaire de Zootechnie de l'I.N.A.-P.G. sur un ensemble de 224 observations individuelles dont 187 effectuées sur des chèvres en lactation et incluant 31 régimes différents.

Les variations de la digestibilité de la matière organique (dM.O.) sont mieux expliquées par le système analytique de Van Soest (N.D.F., A.D.F., A.D.L.) que par celui de Weende (CB), puisque les écarts-types résiduels sont respectivement de 2.3 et 32 points de dM.O. En effet, alors que les différents paramètres d'estimation de la paroi végétale ont des potentiels de prédiction intra-régime équivalents, la lignine (A.D.L.) se distingue des autres par une meilleure prise en compte des différences inter-régimes. La nature botanique du régime permet de prendre en compte une partie des variations de la dM.O. non expliquées par les paramètres de l'analyse chimique. Ainsi, l'introduction du pourcentage de céréales améliore-t-elle nettement la précision de la prédiction de la dM.O. dans le cas de la méthoje de Weende. Le gain de précision obtenu dans le cas du système de Van Soest est beauzoup plus faible, confirmant ainsi l'intérêt que présente ce système pour prendre en compte les différences de digestibilité liées à la nature botanique de la ration.

Aucun effet statistiquement significatif du niveau al'mentaire n'a pu être mis en évidence avec des animaux alimentés au prorata de leur niveau de production. Les équations proposées permettent d'obtenir une précision de l'ordre de l'incertitude couramment admise pour des mesures expérimentales de la digestibilité in vivo.
\end{abstract}

Mots clés : Digestibilité, rations mixtes, ruminant laitier, analyse chimique, prévision.

\section{Introduction}

La digestibilité de la matière organique (dM.O.) d'une ration mixte distribuée à un ruminant laitier conditionne dans une large mesure sa valeur énergétique. Il est donc important d'en connaître les principaux facteurs de variation (nature et compo-

(*) Texte d'une communication présentée lors du séminaire F.A.O. caprins de Grangeneuve 
sition chimique de la ration, niveau alimentaire...) ainsi que de pouvoir les hiérarchiser et les modéliser.

Les modèles de prévision de la digestibilité de la matière organique des fourrages proposés en France présentent un degré de précision satisfaisant (les $4 / 5$ des valeurs des écarts-types résiduels sont inférieurs au seuil de 3,5 p. 100 de dM.O.). Ces modèles utilisent les résultats du schéma analytique de WEENDE et sont spécifiques des différentes espèces végétales considérées à des cycles déterminés (INRA, 1981). Le problème de la prévision de la dM.O. d'un mélange d'aliments a été abordé dans le contexte des aliments composés (Demarquilly, Andrieu, Sauvant, 1978) et, dans ce cas, le fractionnement de VAN SOEST permet d'obtenir une certaine amélioration de la précision de la prédiction de la dM.O. (SAuvant, 1981).

A notre connaissance, aucune méthode n'a été jusqu'alors proposée en France pour prévoir la dM.O. d'une ration mixte sur la base de ses caractéristiques analytiques moyennes. En outre, la bibliographie indique que cette démarche n'a été que rarement appliquée dans les pays étrangers (PAquay, DE BAERE \& Lousse, 1966). L'intérêt d'une telle approche est actuellement renforcée par le développement que connaît le mode de distribution des aliments en "rations complètes".

\section{Matériel et méthodes}

La présente étude a été effectuée à partir de la banque de données des mesures de digestibilité du Laboratoire (INRA) de la Chaire de Zootechnie de l'I.N.A.-P.G. Les animaux utilisés sont des chèvres laitières de race alpine chamoisée ou Saanen. Chaque observation correspond à une mesure individuelle de bilan digestif concernant un même animal recevant, pendant une période de cinq jours consécutifs, une ration mixte, c'est-à-dire au moins deux aliments dont un fourrage. Dans les données considérées, seize aliments concentrés composés ou sous-produits agro-industriels ont été associés à du foin de luzerne ou à de l'ensilage de maïs, ce qui correspond à 31 régimes différents, c'est-à-dire 31 combinaisons d'un aliment avec un autre.

Sur l'ensemble des 224 observations, 37 ont été faites sur des chèvres taries ou en gestation, et le reste sur des chèvres en production. Les aliments concentrés ont été distribués aux animaux en fonction de leur besoin à un rythme moyen d'environ $350 \mathrm{~g} \mathrm{M.S./kg}$ de lait au-dessus de la couverture des besoins d'entretien et de production permise par la ration de base. Lorsque le taux de refus d'un fourrage a dépassé 5 p. 100 (75 p. 100 des cas), il a été procédé, soit à une analyse individuelle du refus, soit à la détermination du pourcentage des différentes fractions du fourrage refusé par l'animal et à l'analyse de chacune de ces fractions afin de calculer la composition analytique du refus pour chaque chèvre.

Les teneurs des constituants suivants ont été déterminées sur les aliments distribués, refusés et les fécès :

- matière sèche et cendres ;

- cellulose brute (AFNOR, 1981);

- paroi végétale ou N.D.F. de VAN SoEST \& Wine (1967) adaptée par Giger et al. (1979) ; 
- lignocellulose ou A.D.F. et lignine sulfurique ou A.D.L. (VAN SoEst, 1963) adaptée pour les aliments concentrés par Giger et al. (1979). L'hydrolyse au détergent acide qui permet d'obtenir le résidu A.D.F. a été effectuée sur le résidu N.D.F., afin d'éliminer les interférences liées aux substances pectiques (BAILEY \& UlyatT, 1970) ;

- azote Kjeldahl.

Les résultats ont été traités par la méthode d'analyses en composantcs principales normées (A.C.P.) décrite notamment par Lebart \& Fenelon (1971). Cette méthode permet d'apprécier de manière descriptive les corrélations entre les différentes variables. Une autre méthode utilisée est l'analyse de co-variance (programme de Seebeck) qui a permis de faire des régressions progressives ascendantes. Le programme d'analyse discriminante (Manova) permet de faire la part des corrélations inter et intrarégimes.

\section{Tableau 1}

Principales caractéristiques des rations considérées*.

Main characteristics of the diets.

\begin{tabular}{|c|c|c|c|c|}
\hline & $\begin{array}{l}\text { Moyenne } \\
\text { Mean }\end{array}$ & $\begin{array}{l}\text { Ecart-type } \\
\text { Standard- } \\
\text { deviation }\end{array}$ & $\begin{array}{l}\text { Minimum } \\
\text { Minimum }\end{array}$ & $\begin{array}{l}\text { Maximum } \\
\text { Maximum }\end{array}$ \\
\hline \multicolumn{5}{|l|}{ Composition chimique (\% MO) } \\
\hline $\begin{array}{l}\text { Chemical composition }(\% \text { OM) } \\
\text { Cellulose brute (CB) } \ldots \ldots \ldots \ldots \\
\text { Crude fibre (CF) }\end{array}$ & 22,6 & 4,5 & 11,6 & 33,1 \\
\hline $\begin{array}{l}\text { Matière azotées totales (MAT) .. } \\
\text { Crude protein }(C P)\end{array}$ & 19,3 & 3,4 & 10,9 & 29,9 \\
\hline NDF $-N D F \ldots \ldots \ldots$ & 44,0 & 7,6 & 24,0 & 63,4 \\
\hline $\mathrm{ADF}-A D F \ldots \ldots \ldots$ & 25,6 & 5,0 & 13,0 & 36,5 \\
\hline $\mathrm{ADL}-A D L$ & 4,9 & 1,6 & 1,4 & 8,2 \\
\hline \multicolumn{5}{|l|}{$\begin{array}{l}\text { Quantités ingérées }\left(\mathrm{g} / \mathrm{kg} \mathrm{P}^{0,75}\right) \\
\text { Intake }\left(\mathrm{g} / \mathrm{kg} W^{0.75}\right)\end{array}$} \\
\hline $\begin{array}{l}\text { Matière sèche (MSI) } \\
\text { Dry matter (DMI) }\end{array}$ & 95,3 & 27,8 & 42,6 & 164,0 \\
\hline $\begin{array}{l}\text { Matière organique digestible } \\
\quad(\text { MOD) } \ldots \ldots \ldots \ldots \ldots \ldots\end{array}$ & 61,5 & 18,7 & 24,3 & 103,2 \\
\hline $\begin{array}{l}\text { Digestible organic matter (DOMI) } \\
\text { Matière organique non digestible } \\
\text { (MOND) } \ldots \ldots \ldots \ldots \ldots \ldots \ldots\end{array}$ & 26,0 & 7,7 & 8,5 & 49,3 \\
\hline $\begin{array}{l}\text { Non digestible organic matter } \\
\text { (NDOMI) }\end{array}$ & & & & \\
\hline $\begin{array}{l}\text { Digestibilité de la matière organique } \\
\quad(\text { DMO) } \ldots \ldots \ldots \ldots \ldots \ldots \ldots\end{array}$ & 70,1 & 3,9 & 60,6 & 79,6 \\
\hline Organic matter digestibility (OMD) & & & & \\
\hline
\end{tabular}

* 224 observations - 224 data. 


\section{Résultats}

\section{A. Caractéristiques générales des rations étudiées}

Le tableau 1 rapporte les paramètres statistiques concernant les teneurs en matières azotées totales, cellulose brute, N.D.F., A.D.F. et A.D. Lignine des régimes étudiés. Ces teneurs sont régulièrement réparties entre les valeurs extrêmes observées (fig. 1, 2, 3). Les caractéristiques analytiques moyennes des 31 régimes sont rapportées dans le tableau 2 ainsi que les proportions de la matière sèche de la ration sous forme d'aliment concentré ou de céréales. Ce dernier terme regroupe les céréales au sens strict, leurs sous-produits (enveloppes) et l'ensilage de maïs. La proportion moyenne d'aliment concentré a été de 39,3 p. 100 ; elle a varié de 0 (régime ensilage de maïs + foin de luzerne) à 64,9 p. 100 . La part des céréales a varié de 0 à 91,7 p. 100 ; cette large variabilité s'explique par la présence, ou non, d'ensilage de maïs dans la

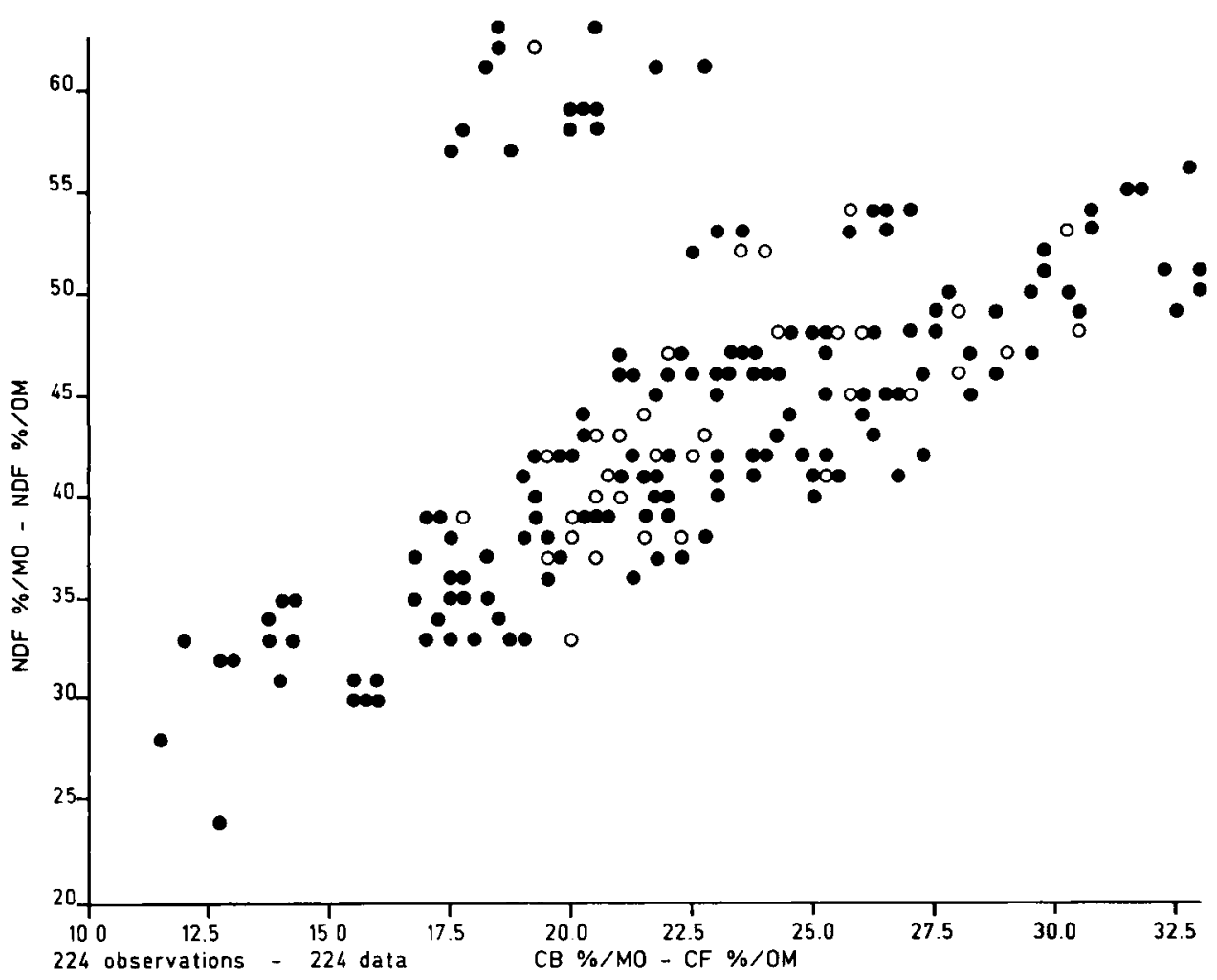

Fig. 1

Relation entre les teneurs en NDF et cellulose brute.

Relationship betweeen NDF and crude fibre values.

Donnée simple (single data).

Données multiples (mixed data). 


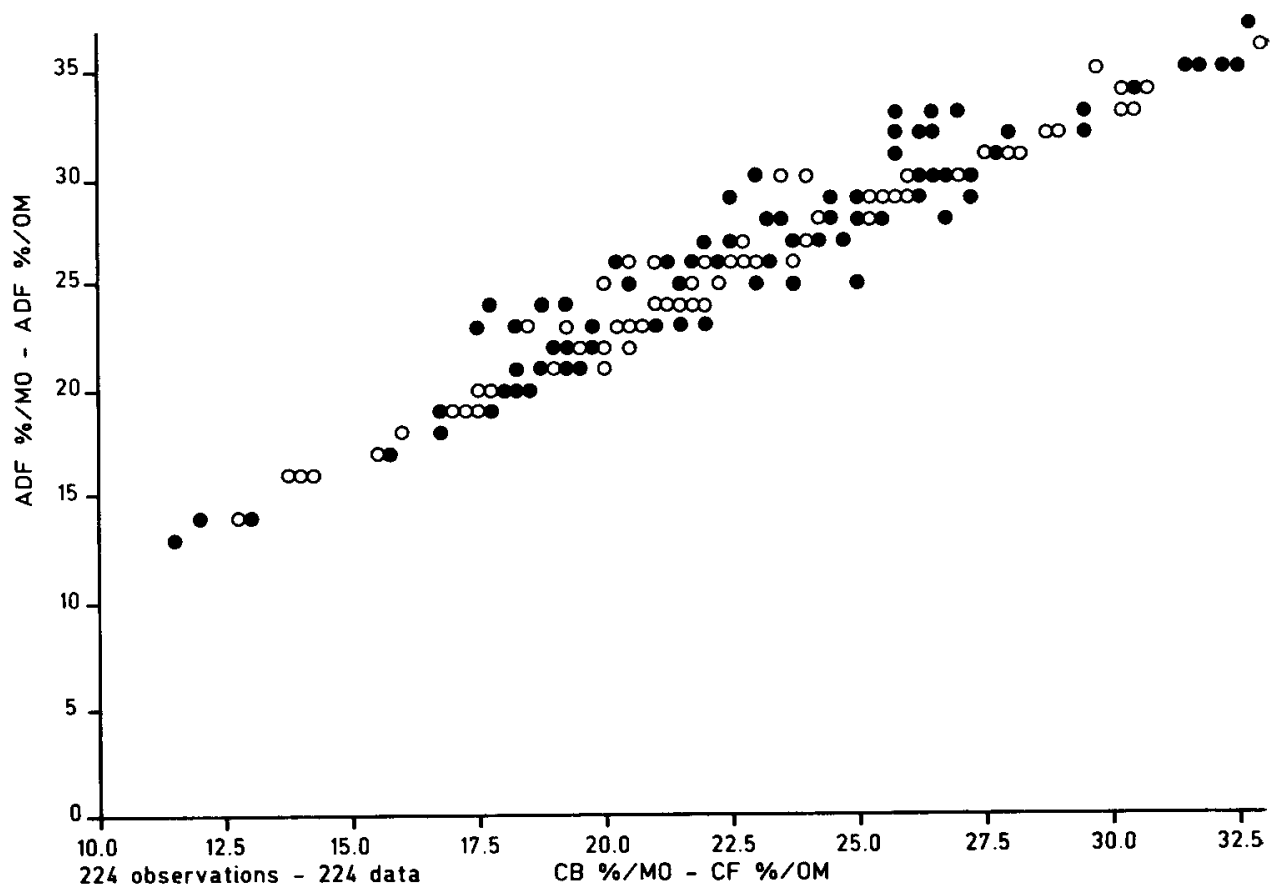

FIG. 2

Relation entre les teneurs en $A D F$ et cellulose brute.

Relationship between $A D F$ and crude fibre values.

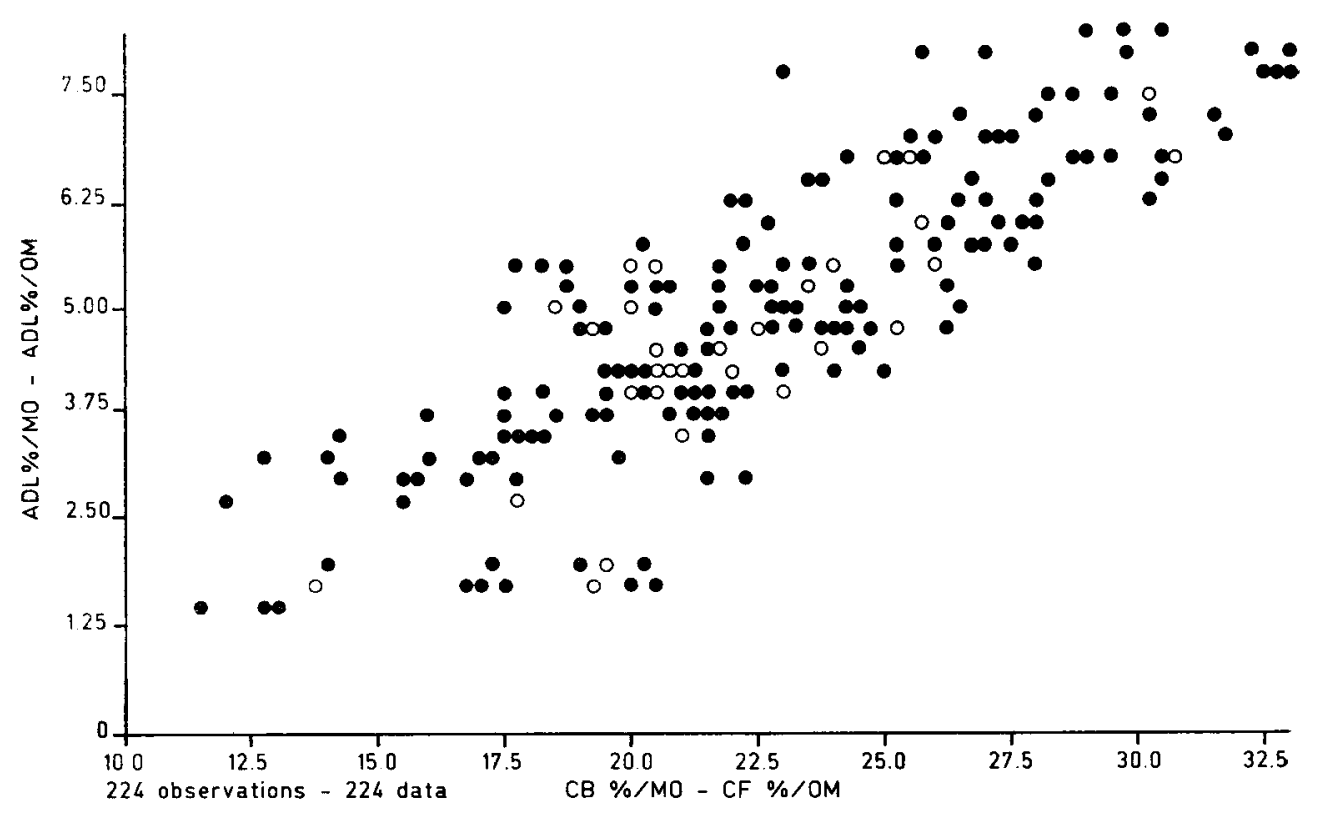

Fig. 3

Relation entre les teneurs en lignine (ADL) et cellulose brute. Relationship between lignin $(A D L)$ and crude fibre values. 
Tableau 2

Principales caractéristiques des différents régimes étudiés.

Mean characteristics of the diets.

\begin{tabular}{|c|c|c|c|c|c|c|c|c|c|c|}
\hline $\begin{array}{c}\text { Régime } \\
\text { de base } \\
\text { Basal } \\
\text { diet }\end{array}$ & $\begin{array}{c}\mathrm{N}^{\circ} \\
\text { régime } \\
N^{\circ} \text { of diet }\end{array}$ & $\begin{array}{c}\text { Nombre } \\
\text { d'obser- } \\
\text { vations } \\
\text { Number } \\
\text { of obser- } \\
\text { vations }\end{array}$ & $\begin{array}{l}\text { DMO } \\
O M D\end{array}$ & $\begin{array}{l}\mathrm{CB} \\
C F\end{array}$ & $\begin{array}{l}\text { NDF } \\
N D F\end{array}$ & $\begin{array}{l}\text { ADF } \\
A D F\end{array}$ & $\begin{array}{l}\text { ADL } \\
A D L\end{array}$ & $\begin{array}{c}\text { MAT } \\
C P\end{array}$ & $\begin{array}{c}\% \\
\text { Concentré } \\
\text { (/MS) } \\
\% \text { Con- } \\
\text { centrate } \\
(/ D M)\end{array}$ & $\begin{array}{c}\% \\
\text { Céréales } \\
\text { (/MS) } \\
\% \\
\text { Cereals } \\
(/ D M)\end{array}$ \\
\hline E & 1 & 2 & 75.5 & 13,1 & 33,7 & 14,9 & 2,8 & 15,0 & 43,5 & 91,7 \\
\hline $\mathrm{E}$ & 2 & 9 & 73,8 & 14,4 & 34,2 & 16,2 & 1.7 & 15,3 & 40,3 & 89,1 \\
\hline $\mathbf{F}, \mathrm{E}, \mathrm{D}$ & 3 & 8 & 64.9 & 19,4 & 58,0 & 24,3 & 5,4 & 25,2 & 59,5 & 85,5 \\
\hline $\mathbf{F}, \mathrm{E}, \mathbf{D}$ & 4 & 8 & 65,4 & 19,8 & 62,0 & 24,3 & 5,0 & 26,8 & 64,9 & 82,8 \\
\hline $\mathrm{E}$ & 5 & 9 & 73.2 & 19,4 & 41,6 & 21,6 & 1,9 & 15,8 & 38,3 & 61,7 \\
\hline $\mathrm{F}, \mathrm{E}$ & 6 & 11 & 69,8 & 20,6 & 408 & 23,1 & 4,4 & 16,8 & 30,6 & 54,8 \\
\hline $\mathrm{F}, \mathrm{E}$ & 7 & 10 & 72,0 & 19,1 & 36,8 & 20,8 & 3,7 & 18,0 & 29,7 & 52,4 \\
\hline$F, E$ & 8 & 6 & 70,6 & 21,2 & 43,4 & 24,1 & 3,8 & 15,2 & 15,9 & 51,3 \\
\hline E & 9 & 2 & 76,8 & 17,7 & 38,6 & 20,2 & 2,7 & 17,1 & 49,2 & 50,8 \\
\hline $\mathrm{F}, \mathrm{E}$ & 10 & 6 & 71,1 & 21,6 & 40,4 & 23,4 & 4,1 & 16,4 & 19,9 & 49,4 \\
\hline $\mathrm{F}, \mathrm{E}$ & 11 & 6 & 70.4 & 22,8 & 44,3 & 25,7 & 4,6 & 17,1 & 19,4 & 48,0 \\
\hline $\mathrm{F}, \mathrm{E}$ & 12 & 8 & 73,1 & 20,9 & 39,8 & 23,5 & 4,4 & 26,7 & 52.2 & 44,0 \\
\hline $\mathrm{F}$ & 13 & 12 & 72,1 & 18,0 & 33,6 & 19,8 & 3,7 & 21,9 & 57,7 & 39.2 \\
\hline F & 14 & 2 & 69,1 & 20,3 & 38.2 & 22,1 & 5,2 & 20,4 & 47,9 & 38,8 \\
\hline$F$ & 15 & 9 & 704 & 19,1 & 36,3 & 21,4 & 4,6 & 17,8 & 51,4 & 38,6 \\
\hline$F, E$ & 16 & 8 & 62,9 & 31,1 & 54.2 & 34,5 & 7,1 & 13,4 & 0,0 & 34,5 \\
\hline $\mathrm{F}, \mathrm{E}$ & 17 & 10 & 71,0 & 22,7 & 46,4 & 27,0 & 4,6 & 16,5 & 26,5 & 33,6 \\
\hline$F$ & 18 & 5 & 72.4 & 20,8 & 34,5 & 22,4 & 5,3 & 21,2 & 44,4 & 33,3 \\
\hline $\mathrm{F}, \mathrm{E}$ & 19 & 12 & 71,9 & 23,0 & 41,8 & 25,6 & 4,2 & 18,7 & 29,4 & 32,6 \\
\hline $\mathrm{F}$ & 20 & 2 & 70.6 & 22,5 & 37,6 & 25,2 & 5,9 & 21,1 & 45,1 & 30,7 \\
\hline $\mathrm{F}$ & 21 & 4 & 69,9 & 22,7 & 37,4 & 24,9 & 6,1 & 20,9 & 36,8 & 27,6 \\
\hline $\mathrm{F}$ & 22 & 18 & 65.7 & 26,7 & 48,5 & 30,2 & 7,0 & 20,3 & 36,7 & 22,8 \\
\hline$F, E$ & 23 & 2 & 68,7 & 26,8 & 42,8 & 29,8 & 7,2 & 20,1 & 33,9 & 19,2 \\
\hline$F$ & 24 & 5 & 63,6 & 27,1 & 45,4 & 30,1 & 9,1 & 23,2 & 43,8 & 13,1 \\
\hline $\mathrm{F}$ & 25 & 18 & 70,1 & 26,8 & 45,3 & 29,9 & 5,9 & 19,9 & 37,5 & 6,8 \\
\hline$F$ & 26 & 5 & 74,0 & 24,8 & 41,4 & 27,7 & 4,4 & 21,3 & 54,0 & 0,0 \\
\hline F & 27 & 12 & 72,3 & 24,6 & 53,1 & 30,7 & 5,7 & 19,6 & 52,4 & 0,0 \\
\hline F & 28 & 2 & 71,1 & 26,1 & 53,6 & 33,4 & 7,0 & 19,0 & 52,3 & 0,0 \\
\hline F & 29 & 2 & 73,1 & 24,4 & 40,6 & 25,4 & 4,4 & 22,5 & 47,3 & 0,0 \\
\hline$F$ & 30 & 9 & 69,1 & 27,0 & 47,5 & 30,3 & 5,5 & 18,2 & 45,6 & 0,0 \\
\hline $\mathrm{F}$ & 31 & 2 & $67: 0$ & 29,8 & 51,7 & 34,9 & 8,1 & 19,8 & 33,7 & 0,0 \\
\hline
\end{tabular}

Les données analytiques sont exprimées en p. 100 de la matière sèche.

Analytical data are expressed as p. 100 of dry matter.

Nature de la ration de base - Nature of basal diet :

D : drèches de brasserie - brewers grain.

$\mathrm{E}$ : ensilage de maïs - maize silage.

$\mathrm{F}$ : foin de luzerne - lucerne hay. 
ration et par la proportion de grains et d'enveloppes de céréales dans les aliments concentrés qui a, en effet, varié de 0 à 100 p. 100.

Les quantités ingérées mesurées ont varié entre 42,6 et 164,0 g M.S./ $\mathrm{kg}$ P0,75 (tabl. 1). Elles se répartissent en deux groupes distincts : d'une part les animaux en gestation ou taris (niveau moyen : $55,7 \mathrm{~g} \mathrm{M} . \mathrm{S} . / \mathrm{kg}^{\mathbf{0}, 75}$ ), et d'autre part, les animaux en production (niveau moyen : $103,1 \mathrm{~g} / \mathrm{kg} \mathbf{P}^{0,75}$ ). Les digestibilités mesurées de la matière organique se répartissent selon une loi normale dans un intervalle de 20 points environ, de 60,6 à 79,6 p. 100 (fig. 4).

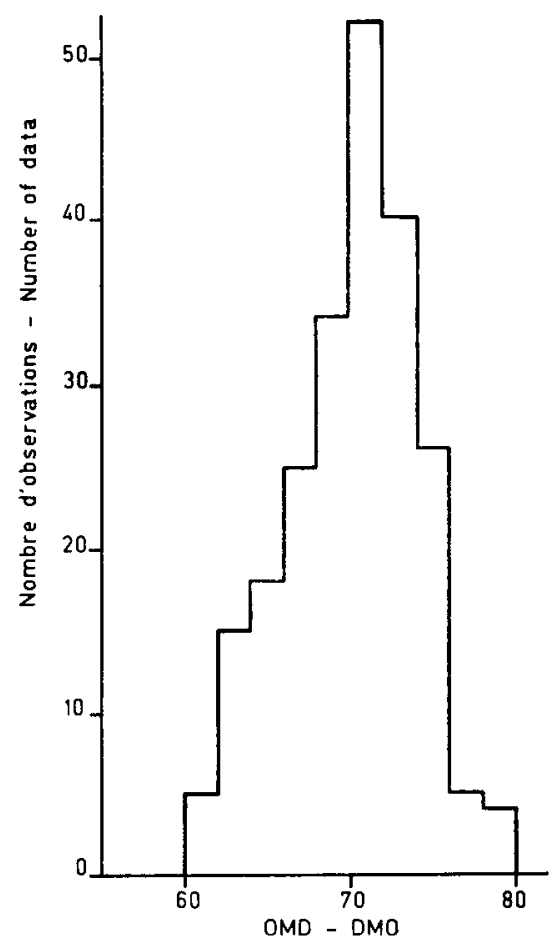

FIG. 4

Histogramme des valeurs de digestibilité de la matière organique.

Histogramm of organic matter digestibility values.

B. Etude descriptive de l'ensemble des données

Pour avoir une vue à la fois précise et synthétique de l'ensemble des données considérées et pour analyser les interrelations entre les différentes caractéristiques chimiques des rations, une analyse en composantes principales a été appliquée aux 224 observations caractérisées par 13 critères, les 9 du tableau 1 complétées par les rapports A.D.L./A.D.F. et A.D.L./N.D.F. ainsi que les proportions de foin et d'en- 
silage de maïs dans le régime qui sont prises comme variables supplémentaires (variables sans poids).

Les deux premières composantes rendent compte de 71 p. 100 de la variation, la figure 5 traduit les corrélations entre les 13 variables et les deux premières composantes et les valeurs des projections des barycentres des régimes. La première composante exprime la relation générale inverse qui associe la digestibilité de la matière organique et les teneurs en constituants pariétaux des régimes. La seconde composante traduit les variations de la quantité ingérée qui apparaissent être globalement indépendantes de celles de la digestibilité et de la teneur en constituants pariétaux.

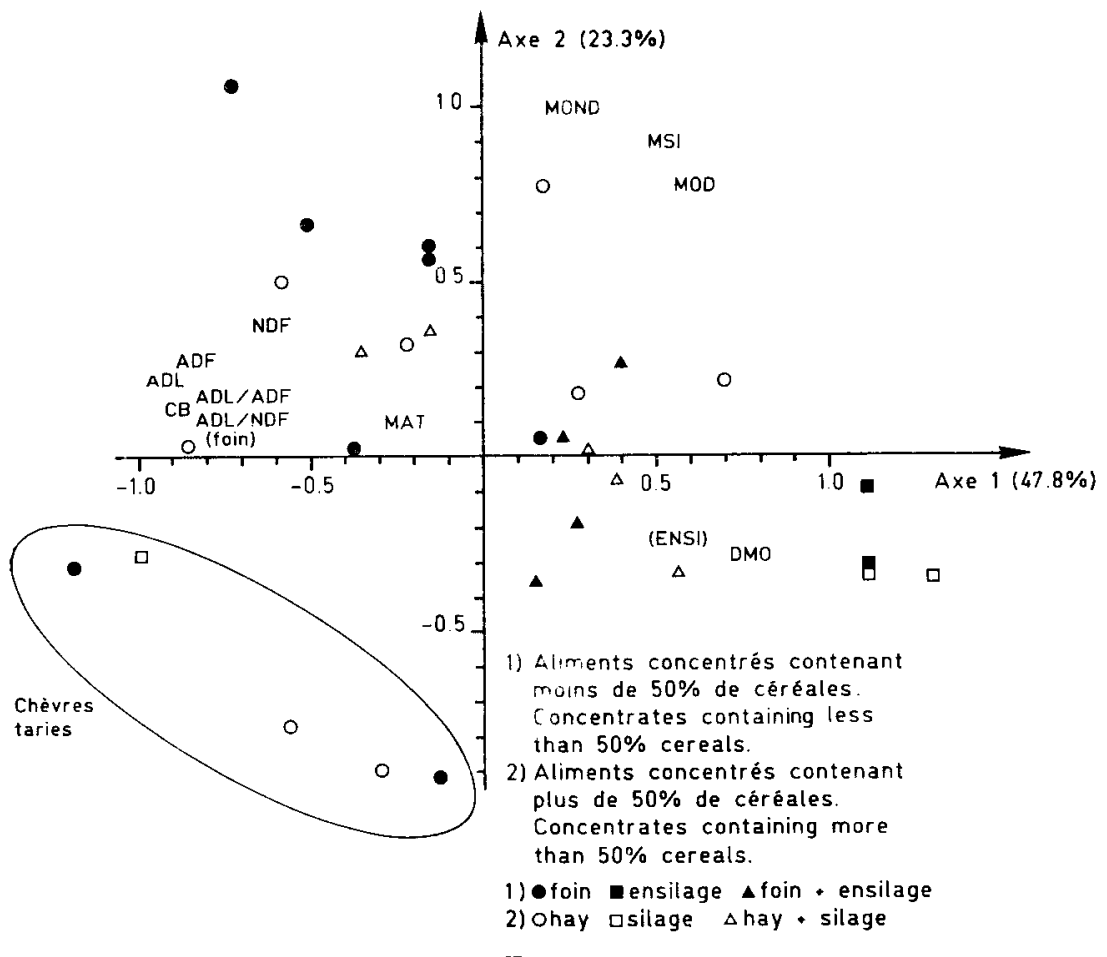

FIG. 5

Projection des variables et des barycentres des régimes sur les composantes 1 et 2 dans l'ACP. Projection of variates and diet barycenters on the components 1 and 2 in the PCA.

Le nuage des projections des régimes est relativement homogène, en particulier les régimes des animaux taris ne se discriminent pas sur la première composante de certains régimes distribués à des animaux en lactation. La nature du fourrage explique une partie de la variation associée à la première composante.

La troisième composante principale explique 12,9 p. 100 de la variation des données (fig. 6). Elle traduit, notamment, les différences de degré de lignification des parois végétales des régimes. La nature et le pourcentage du fourrage, mais aussi 
ceux de l'aliment concentré (concentré riche en amidon ou riche en paroi végétale facilement digestible), sont à l'origine des variations du degré de lignification des régimes considérés.

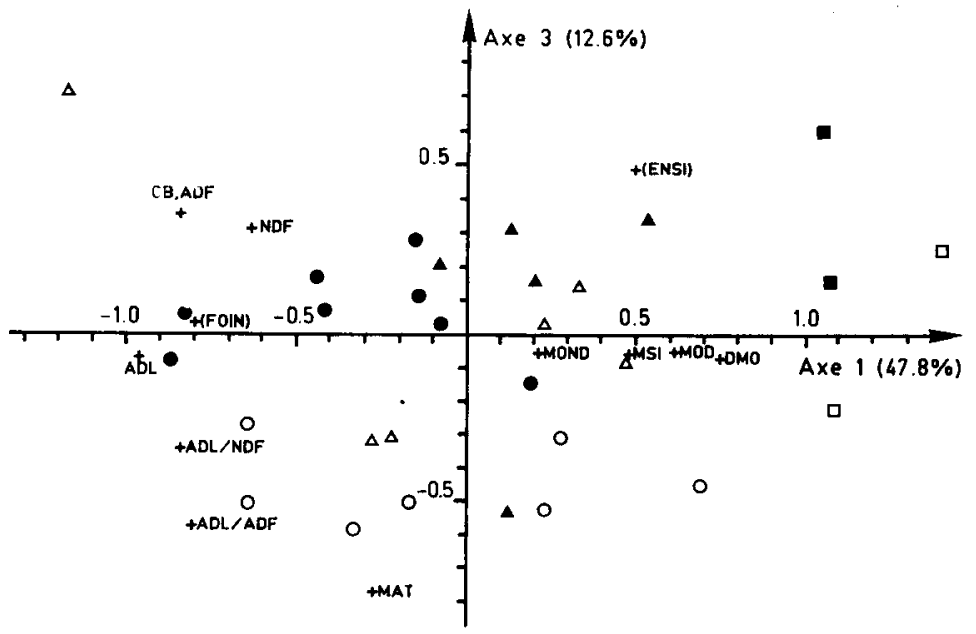

FIG. 6

Projection des variables et des barycentres des régimes sur les composantes 1 et 3 dans l'ACP. Projection of variates and diet barycenters on the components 1 and 3 in the PCA.

\section{Relations entre les différents critères associés à la paroi végétale (1)}

Les teneurs en cellulose brute, N.D.F., A.D.F. et A.D.L. sont significativement et positivement corrélées entre elles (tabl. 3). Le coefficient de corrélation le plus élevé $(0,97)$ associe les teneurs en cellulose brute et A.D.F. et le plus faible $(0,59)$, les teneurs en N.D.F. et A.D.L. Dans le but de comparer la teneur du résidu pariétal selon la méthode de Weende (cellulose brute) à celles issues des méthodes Van Soest, les relations associant chacun des critères de paroi Van Soest à la teneur en cellulose brute ont été étudiées.

La figure 1 traduit la relation générale entre les teneurs en N.D.F. et cellulose brute des rations étudiées :

$$
\text { N.D.F. }=22,7+\underset{(0,09)}{0,95} \text { C.B. } \quad(\text { R. }=0,56, \mathrm{n}=224, \text { E.T.R. }=6,33)
$$

Cette figure met en évidence que deux régimes se distinguent des 29 autres pour cette relation : les régimes 3 et 4 qui contiennent en moyenne 60 p. 100 de drèches de brasserie présentent une teneur en N.D.F. supérieure d'environ 20 points à la régres-

(1) Dans les équations les composants sont exprimés en p. 100 M.O. 


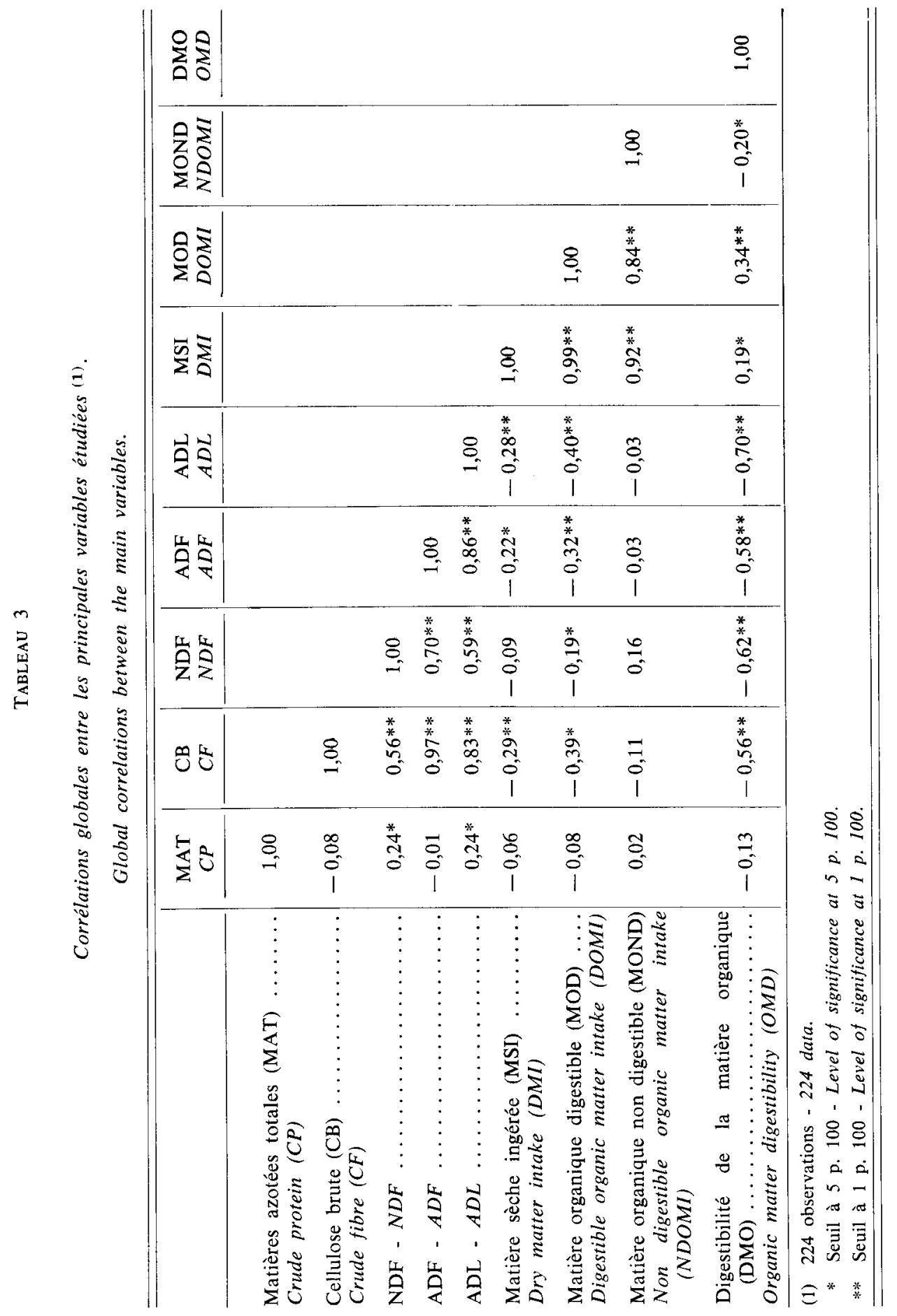


sion calculée. Ce fait explique largement l'importance de l'écart-type résiduel de la régression; en effet, lorsque ces deux régimes sont exclus du calcul, la relation devient :

$$
\text { N.D.F. }=16,1+\underset{(0,05)}{1,17} \text { C.B. } \quad(\text { R. }=0,84, \mathrm{n}=208, \text { E.T.R. }=3,49)
$$

La comparaison des coefficients de corrélation entre le N.D.F. et la C.B. aux niveaux global $(0,59)$, inter-régime $(0,52)$ et intra-régime $(0,83)$ montre également l'effet du régime sur la relation N.D.F. - C.B.

Les teneurs en A.D.F. et cellulose brute sont étroitement associées selon la relation (fig. 2) :

$$
\text { A.D.F. }=1,09+\underset{(0,02)}{1,09} \text { C.B. } \quad(\text { R. }=0,97, \mathrm{n}=224 \text {, E.T.R. }=1,25)
$$

La valeur des coefficients de régression indiquent que la teneur en A.D.F. est légèrement supérieure à la teneur en cellulose brute.

La figure 3 traduit la relation associant les teneurs en A.D. Lignine et cellulose brute des rations étudiées :

$$
\text { A.D.L. }=-1,80+\underset{(0,01)}{0,30 \text { C.B. }} \quad(\text { R. }=0,83, n=224, \text { E.T.R. }=0,89)
$$

La corrélation intra-régime C.B.-A.D.L. est de 0,90, c'est-à-dire supérieure aux corrélations globale et inter-régime $(0,83)$, ce qui montre l'influence de la nature du régime sur la relation considérée.

\section{Modèles de prévision de la digestibilité de la matière organique des rations mixtes}

\section{A partir des caractéristiques analytiques}

Le tableau 4 montre que la teneur en A.D. Lignine est la caractéristique analytique individuelle la mieux associée aux variations de la digestibilité des rations $(\simeq 50$ p. 100 de la variance expliquée et un écart-type résiduel de 2,8 points).

$$
\begin{aligned}
& \text { D.M.O. }=78,5-1,71 \text { A.D.L. } \quad(\text { R. }=0,71, \mathrm{n}=224 \text {, E.T.R. }=2,8) \\
& (0,12)
\end{aligned}
$$

Les trois autres caractéristiques de la paroi présentent des potentiels de prévision assez comparables avec un peu plus du tiers de variance commune avec la digestibilité, soit des E.T.R. de 3,0 à 3,2 points.

Lorsque les corrélations globales sont éclatées en corrélations inter et intrarégimes, on observe qu'à l'intérieur d'un même régime, les potentiels de prédiction des teneurs en cellulose brute, N.D.F., A.D.F. sont très comparables et s'améliorent par rapport aux relations globales, la lignine conservant le même potentiel de prédiction (tabl. 5). Lorsque les moyennes de chaque régime sont considérées, ce qui correspond aux corrélations inter-régimes, la teneur en A.D.L. est aussi un prédicteur supérieur aux trois autres. 


\section{TABLEAU 4}

Relations entre la digestibilité de la matière organique et différents critères analytiques.

Relationships between organic matter digestibility and different analytical criteria. (224 observations - 224 data).

\begin{tabular}{|c|c|c|c|c|c|c|c|}
\hline \multirow{2}{*}{$\begin{array}{l}\text { Constantes } \\
\text { Constants }\end{array}$} & \multicolumn{7}{|c|}{ Variables explicatives - Determining variables } \\
\hline & $\begin{array}{l}\mathrm{CB} \\
\mathrm{CF}\end{array}$ & $\begin{array}{l}\text { NDF } \\
N D F\end{array}$ & $\begin{array}{l}\mathrm{ADF} \\
A D F\end{array}$ & $\begin{array}{l}\text { ADL } \\
A D L\end{array}$ & $\begin{array}{c}\text { MAT } \\
C P\end{array}$ & $\begin{array}{l}\mathrm{R} \\
R\end{array}$ & $\begin{array}{l}\text { ETR } \\
R S D\end{array}$ \\
\hline 81,2 & $\begin{array}{r}-0,493 \\
(0,048)\end{array}$ & & & & & 0,57 & 3,2 \\
\hline 85,4 & $\begin{array}{r}-0,505 \\
(0,047)\end{array}$ & & & & $\begin{array}{r}-0,202 \\
(0,062)\end{array}$ & 0,59 & 3,2 \\
\hline 84,3 & & $\begin{array}{c}0,323 \\
(0,027)\end{array}$ & & & N.S. & 0,63 & 3,0 \\
\hline 81,6 & & & $\begin{array}{r}-0,449 \\
(0,042)\end{array}$ & & & 0,58 & 3,1 \\
\hline 84,5 & & & $\begin{array}{r}-0,450 \\
(0,042)\end{array}$ & & $\begin{array}{r}-0,152 \\
(0,062)\end{array}$ & 0,60 & 3,1 \\
\hline 78,5 & & & & $\begin{array}{r}-1,713 \\
(0,116)\end{array}$ & N.S. & 0,71 & 2,8 \\
\hline 83,6 & & $\begin{array}{r}-0,169 \\
(0,028)\end{array}$ & & $\begin{array}{r}-1,243 \\
(0,133)\end{array}$ & N.S. & 0,75 & 2,6 \\
\hline 81,6 & & $\begin{array}{r}-0,232 \\
(0,030)\end{array}$ & $\begin{array}{c}0,319 \\
(0,071)\end{array}$ & $\begin{array}{r}-1,923 \\
(0,199)\end{array}$ & & 9,78 & 2,5 \\
\hline 75,9 & & $\begin{array}{r}-0,294 \\
(0,031)\end{array}$ & $\begin{array}{c}0,536 \\
(0,080)\end{array}$ & $\begin{array}{r}-2,477 \\
(0,218)\end{array}$ & $\begin{array}{c}0,288 \\
(0,057)\end{array}$ & 0,80 & 2,3 \\
\hline
\end{tabular}

() Ecart-type du coefficient.

Standard deviation of the coefficient.

L'association de la teneur en N.D.F. à celle en A.D.L. permet de diminuer de 0,2 point l'écart-type résiduel de la régression explicative des variations de la dM.O. L'A.D.F. a une influence significative propre, ce qui permet à l'association des trois critères analytiques (N.D.F., A.D.F., A.D.L.) d'expliquer environ 60 p. 100 de la variance de la dM.O. avec un écart-type résiduel de 2,5 points.

L'influence de la teneur en matières azotées totales sur la dM.O. est faible (cas des modèles avec cellulose brute ou A.D.F. ou N.D.F., A.D.F. et A.D.L.) ou même nulle (modèles avec N.D.F. ou A.D.L.). 


\section{TABLEAU 5}

Coefficients de corrélation entre la digestibilité de la matière organique et les caractéristiques liées aux parois végétales des rations.

Correlation coefficients between organic matter digestibility and cell-wall characteristics of diets.

\begin{tabular}{|c|c|c|c|}
\hline & $\begin{array}{c}\text { Corrélation globale } \\
\text { Global correlation } \\
\text { (224 observations) } \\
\text { (224 data) }\end{array}$ & $\begin{array}{l}\text { Corrélations intra-régime } \\
\text { Within diet correlation }\end{array}$ & $\begin{array}{c}\text { Corrélations inter-régime } \\
\text { Between diet correlation } \\
\text { (31 régimes) } \\
\text { (31 diets })\end{array}$ \\
\hline $\begin{array}{l}\mathrm{CB} \\
\mathrm{CF}\end{array}$ & $-0,56$ & $-0,64$ & $-0,54$ \\
\hline $\begin{array}{l}\text { NDF } \\
\text { NDF }\end{array}$ & $-0,63$ & $-0,65$ & $-0,67$ \\
\hline $\begin{array}{l}\mathrm{ADF} \\
A D F\end{array}$ & $-0,58$ & $-0,66$ & $-0,57$ \\
\hline $\begin{array}{l}\mathrm{ADL} \\
A D L\end{array}$ & $-0,71$ & $-0,71$ & $-0,74$ \\
\hline
\end{tabular}

En définitive, l'association de la teneur en M.A.T. aux critères de Van Soest permet d'expliquer 65 p. 100 de la variance de la digestibilité de la matière organique avec un écart-type résiduel de 2,3 points, soit un gain de 0,9 point par rapport au modèle «cellulose brute et matières azotées totales».

Le niveau alimentaire, caractérisé par la quantité de matière sèche ingérée par kilogramme de poids métabolique, n'a pas d'influence significative spécifique dans les différents modèles rapportés au tableau 4.

2. A partir des caractéristiques analytiques combinées à des caractéristiques botaniques de la ration

Cette partie de l'étude a été effectuée sur les caractéristiques moyennes des régimes (étude inter-régimes). Pour évaluer la part de la variation résiduelle imputable à la nature botanique de la ration, nous avons relié la résiduelle de l'équation interrégimes (dM.O. fonction de la cellulose brute) au pourcentage moyen de céréales dans le régime considéré (fig. 7).

Un examen de ce graphique montre que les régimes incluant un aliment concentré contenant plus de 50 p. 100 de céréales ou enveloppes de céréales (aliments de type 《amidon») présentent pour la majeure partie des valeurs de digestibilité de la matière organique surestimées par le modèle, tandis que l'inverse se produit pour les régimes incluant un aliment concentré contenant peu ou pas de céréales. Etant donné qu'une dizaine d'aliments «amidon» ont été formulés afin d'avoir a priori, une digestibilité de la matière organique égale à celle d'un aliment de type «paroi 


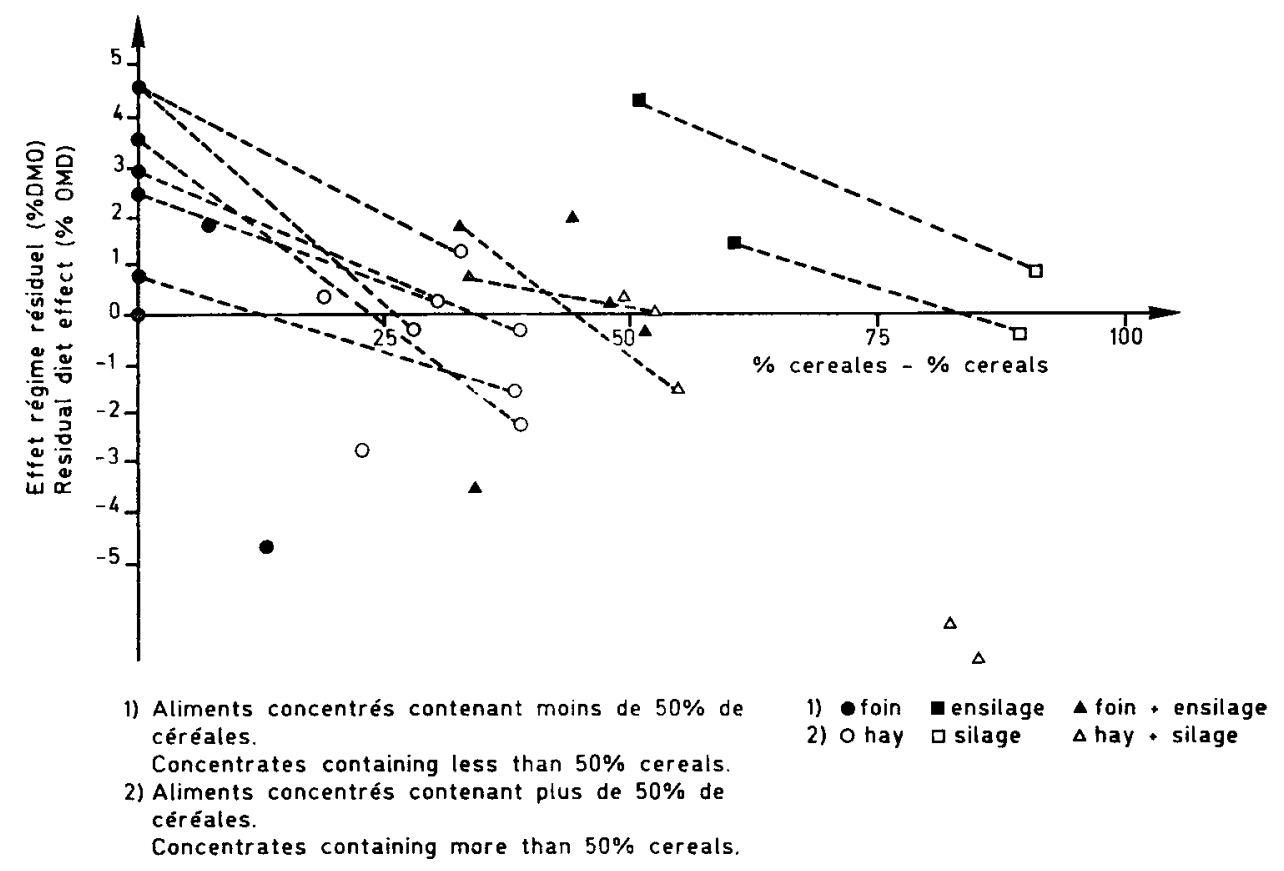

Fig. 7

Relation entre le pourcentage de céréales dans la ration et l'effet régime dans l'équation reliant la DMO à $C B$ et MAT.

Relationship between cereal percentage in the diet and diet effect in the equation relating $O M D$ to $C F$ and $C P$.

digestible », il est possible de calculer que la différence moyenne séparant les régimes amidon et paroi est de 3,1 points, ce qui est statistiquement différent de zéro au seuil de 5 p. 100. Lorsqu'une comparaison analogue est effectuée en prenant comme critères analytiques les valeurs issues des méthodes de Van Soest, la différence entre les couples amidon et paroi descend à 1,2 point, et ne diffère pas statistiquement de zéro au seuil de 5 p. 100 (fig. 8).

L'étude de ces variations résiduelles incite à prendre en compte le pourcentage de céréales (P.C.) dans la ration au sein des modèles de prédiction de la digestibilité. Cette prise en compte améliore sensiblement la précision dans le cas de la régression avec la teneur en cellulose brute, puisque l'écart-type résiduel est diminué de 1 point (tabl. 6). Cependant, une étude des variations résiduelles de ce dernier modèle révèle une tendance à sous-estimer la dM.O. des rations contenant de l'ensilage de maïs. Le fait d'éclater le pourcentage de céréales (P.C.) en deux variables qui correspond aux pourcentages de la matière sèche représentés par l'ensilage de maïs (E.M.) et par des céréales et leurs sous-produits apportés par l'aliment concentré (C.E.) permet un nouveau gain de précision $(0,6$ point). Les coefficients de cette dernière équation indiquent que l'ensilage de maïs cause, par point d'incorporation, un biais inférieur aux céréales et à leurs sous-produits (tabl. 6). 


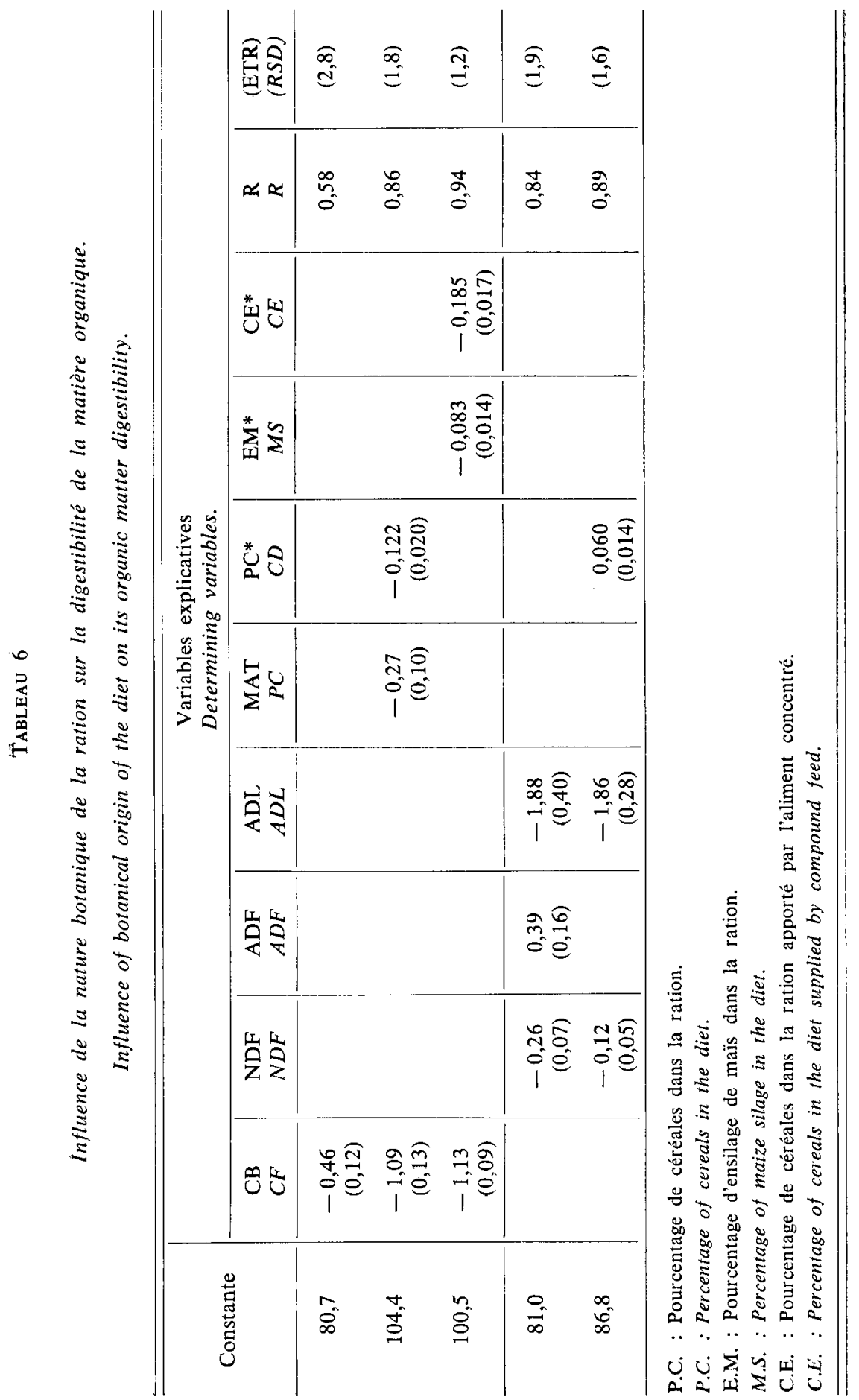




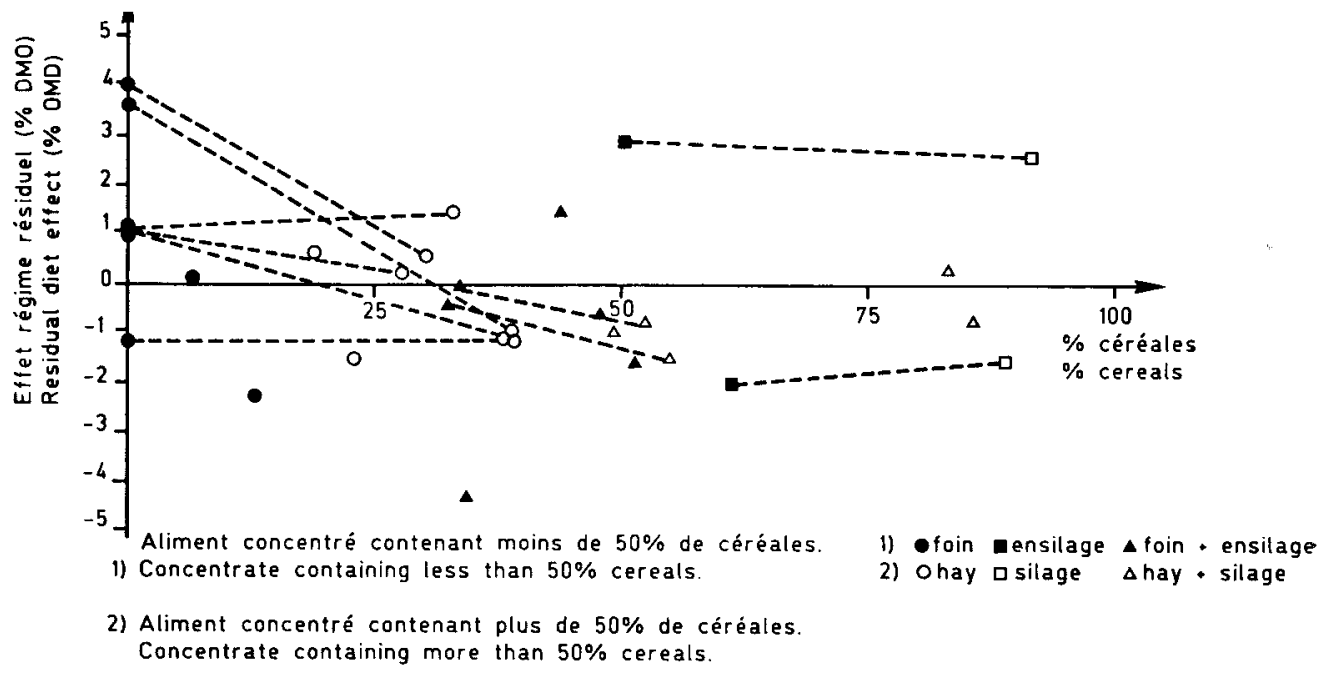

FIG. 8

Relation entre le pourcentage de céréales dans la ration et l'effet régime dans l'équation reliant la $D M O$ à $N D F$ et $A D L$.

Relationship between cereal percentage in the diet and effect in the equation relating $O M D$ function to $N D F$ and $A D L$.

Une approche analogue a été effectuée avec les critères analytiques de Van Soest. Le gain lié à l'introduction du pourcentage de céréales (P.C.) est plus faible que dans le cas précédent $(0,3$ point de dM.O.). Le fait de séparer les céréales en deux fractions, l'ensilage de maïs et le pourcentage de céréales provenant de l'aliment concentré n'améliore ni l'écart-type résiduel, ni le coefficient de corrélation multiple.

Dans la mesure où la teneur en céréales de la ration est hautement corrélée à la teneur en «amidon + sucres » estimée d'après les tables Protector (Protecror, 1980) $(\mathrm{r}=0,83, \mathrm{n}=31)$, nous avons testé l'introduction de ce critère dans les équations liant la dM.O. aux teneurs en paroi végétale estimées soit par la méthode de Weende, soit par les méthodes de Van Soest (N.D.F., A.D.F., A.D.L.). Il n'intervient pas significativement dans les équations considérées et ne permet pas d'expliquer les variations résiduelles dans les équations combinant les critères analytiques et botaniques.

Au niveau de l'ensemble des observations, la prise en compte du pourcentage de céréales dans la ration améliore nettement (baisse de 0,75 point de l'écart-type résiduel) la prévision de la dM.O. avec la méthode de Weende :

$$
\begin{aligned}
& \text { dM.O. = 101,1 - 0,97 C.B. - 0,28 M.A.T. - 0,108 P.C. } \\
& (0,05) \quad(0,05) \quad(0,089) \\
& (\mathrm{R} .=0,78, \mathrm{n}=224 \text {, E.T.R. }=2,5)
\end{aligned}
$$

Par contre, le gain de précision est plus faible (baisse de 0,5 point de E.T.R.) pour les critères analytiques de Van Soest : 


$$
\begin{aligned}
& \text { dM.O. }=90,5 \text { - 0,30 A.D.F. - 1,86 A.D.L. - 0,097 P.C. } \\
& (0,06) \quad(0,17) \quad(0,007) \\
& (\text { R. }=0,85, \mathrm{n}=224 \text {, E.T.R. }=2,1)
\end{aligned}
$$

L'éclatement de ce pourcentage en pourcentage d'ensilage de maïs (E.M.) et pourcentage de céréales (C.E.) dans l'aliment concentré améliore la prédiction dans le cas de la méthode Weende :

$$
\begin{array}{r}
\text { dM.O. }=97,8-\underset{(0,05)}{1,04} \text { C.B. }-\underset{(0,080)}{0,070 \text { E.M. }} \begin{array}{r}
-0,177 \text { C.E. } \\
(0,010)
\end{array} \\
(R .=0,85, \mathrm{n}=224, \text { E.T.R. }=2,1)
\end{array}
$$

et n'apporte par contre pas de gain appréciable de précision dans le schéma de Van Soest :

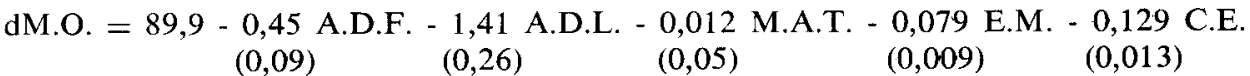

$$
\begin{aligned}
& (\text { R. }=0,86, \mathrm{n}=224, \text { E.T.R. }=2,0 \text { ) }
\end{aligned}
$$

La prise en compte simultanée des pourcentages d'ensilage de maïs et de céréales dans l'aliment permet à la cellulose brute d'avoir un pouvoir prédicteur analogue de la dM.O. à celui de la combinaison de N.D.F. et de l'A.D.L. de Van Soest.

Une autre approche consiste à comparer la digestibilité des rations mixtes à la valeur calculée à partir de la digestibilité des aliments concentrés calculés à partir des valeurs des tables (INRA, 1978) et de celle des fourrages mesurée sur les chèvres puisqu'elles ne digèrent pas le foin comme les moutons (GIGER et al., 1986). La précision obtenue est du même ordre de grandeur que celle issue de la combinaison des critères analytiques et botaniques :

dM.O. mesurée $=0,995$ dM.O. calculée

$$
\text { (R. }=0,85, \mathrm{n}=224, \text { E.T.R. }=2,1)
$$

\section{Discussion}

Les traitements chimiques employés pour déterminer la teneur en cellulose brute (hydrolyses en milieu acide puis basique) conduisent à une élimination importante des hémicelluloses, faible de la cellulose et variable de la lignine (NEHRING \& HofFMANN, 1969). De ce fait, il est logique que la teneur en cellulose brute soit surtout corrélée à la teneur en A.D.F. qui devrait virtuellement renfermer la cellulose et la lignine (Kim, Gillingham \& Loadholt, 1967), mais qui contient aussi des substances comme la cutine ou des protéines dénaturées et qui a perdu une partie de la cellulose et de la lignine (Porter \& Singleton, 1971; Gordon \& Neudoerffer, 1973 ; Theander \& AMAN, 1980 ; JARRIGE, 1981). Le comportement particulier des drèches de brasserie dans la relation entre N.D.F. ou «paroi végétale » et la cellulose brute tient probablement au fait que ces aliments sont riches en matières azotées qui peuvent contaminer le résidu N.D.F., puisque les dosages ont été effectués sans utilisation du sulfite de sodium connu pour son action déprotéinisante mais également délignifiante (VAN SoEst, 1978 ; Giger, 1985). 
Le degré de précision de la prévision de la dM.O. par les différents critères choisis peut être estimé facilement par la valeur de l'écart-type résiduej de la régression. Le taux de lignine sulfurique (ou A.D.L.) est le meilleur prédicteur de la dM.O. au niveau de l'ensemble des données et permet à lui seul d'expliquer la moitié de la variance avec un écart-type résiduel de 2,8 points. Cette supériorité de l'A.D.L. sur les autres critères réside dans la meilleure aptitude de l'A.D.L. à prendre en compte les différences inter-régimes puisque, au niveau de l'ensemble des données, les corrélations inter-régimes les plus élevées correspondent à l'A.D.L., et que la même observation peut-être faite au niveau des relations sur les caractéristiques moyennes des régimes. Au niveau des relations intra-régimes, les différents critères présentent des pouvoirs de prédiction analogues. Ces différences de comportement des critères au niveau des relations inter-régime et intra-régime avaient déjà été observées par SAUVANT (1980) pour les aliments concentrés à partir d'une interprétation statistique des résultats de l'Institut Rowett. Elle peut expliquer que la lignine dont l'estimation est moins précise que celles des autres critères pariétaux, du fait de sa faible teneur, soit un critère moins bon que les autres au niveau des relations intra, mais supérieur aux autres quand plusieurs «types de parois végétales» sont impliqués. Le premier cas correspond aux fourrages, essentiellement représentés par deux familles végétales, donc deux types de parois (Gordon, 1975 ; MAlossini et al., 1973 ; JARRIGE, 1981) et le second au cas présent.

Dans l'étude présente, la teneur en cellulose brute considérée seule donne pour les différentes rations mixtes considérées, une précision aussi bonne ou souvent meilleure que celle généralement observée avec ce critère pour les fourrages (AXELSSON, 1951 ; Walker \& Hepburn, 1955 ; Nehring, 1975; Kirchgessner \& Kellner, 1977 ; Aerts et al., 1977 ; VenCl \& Flam, 1981 ; tables de l'INRA, 1981 ; revue de Minson, 1982), pour les aliments concentrés et sous-produits agro-industriels (NeHRING, 1974, R.R.I., 1975 et 1978 ; AERTs et al., 1977 ; MAlossini et al., 1979 ; Sauvant, 1980) ou par Nordfeldt, Svanberg \& Claesson (1949) ou Laube (1960) pour les rations mixtes. L'ordonnée à l'origine de cette équation $(80,7)$ figure parmi les valeurs les plus faibles des différentes équations considérées $(74,3$ à 106,5) de même que la pente : - 0,46/0,05 à 1,46 . Ces différences s'expliquent par la présence de matières premières à cellulose facilement digestible dans une partie des rations considérées. L'introduction du taux de céréales dans l'équation non seulement améliore significativement l'écart-type résiduel mais également modifie significativement l'ordonnée à l'origine et la pente concernant la teneur en cellulose brute qui augmente de plus du double. Ces deux valeurs ne sont pas modifiées significativement par l'éclatement du pourcentage de céréales en pourcentage d'ensilage de maïs et de céréales provenant du concentré. L'importance de ces modifications met en évidence la liaison entre le type d'équations proposées et la nature des aliments sur lesquels elles ont été obtenues.

La précision observée avec la teneur en lignine (A.D.L.) est proche des valeurs de la littérature (EDWARDS, 1973 ; AERTs et al., 1977), mais inférieure aux valeurs habituellement proposées (GIGER, 1985). La comparaison des écarts-types résiduels obtenus avec les méthodes de Weende et de Van Soest montre que cette dernière est plus précise que la première. Comme les teneurs en A.D.F. et C.B. sont hautement corrélées, la différence du potentiel de prédiction porte sur le N.D.F. et la lignine. L'effet significatif du pourcentage de céréales dans les équations montre que les systèmes ne rendent pas compte fidèlement des différences de digestibilité des parois liées à l'origine botanique des aliments composant la ration. L'absence d'effet du 
critère d'estimation des glucides de réserve (sucre + amidon) qui est cependant hautement corrélé au pourcentage de céréales sous-entend qu'il n'y a pas globalement d'interaction digestive négative entre les fractions pariétales et celles riches en glucides de réserve. Cette absence d'effet global peut s'expliquer par le fait que dans le cas présent la teneur moyenne "sucre + amidon " est de 18,5 p. 100, alors que les phénomènes d'interaction digestive seraient surtout marqués pour des teneurs en glucides de réserve (sucre + amidon) supérieures à 20 p. 100 de la matière sèche totale comme UDEN (1984) l'a observé avec un régime contenant 30 p. 100 d'aliment concentré à base d'orge et d'avoine.

Cependant, dans la mesure où l'introduction du pourcentage de céréales améliore beaucoup la précision au niveau de la méthode de Weende, et peu pour celui de Van Scest, et que l'éclatement de ce pourcentage de céréales en pourcentage de céréales provenant de l'aliment concentré et de celui provenant de l'ensilage de maïs a le même effet, on peut en déduire que la cellulose brute reflète moins bien la valeur nutritive des rations mixtes que les constituants pariétaux de Van Soest car elle est moins apte à prendre en compte les différents types de paroi végétale.

Les écarts-types résiduels obtenus avec les équations faisant intervenir la nature chimique et botanique des aliments sont de l'ordre de 2 points, c'est-à-dire équivalents alux valeurs couramment observées inter- et intra-animal pour une ration donnée (R.R.I., 1975 ; Charlet-Lery, 1969). Ceci montre le degré de fiabilité des équations proposées du fait de la variabilité de la nature des régimes considérés liés à la nature de l'aliment concentré, mais sans oublier cependant que, seuls, deux types de fourrages grossiers, mais très différents, ont été utilisés. Cet écart est équivalent à celui obtenu en utilisant la méthode additive pour les concentrés et les valeurs de digestibilité mesurées pour les fourrages. Cette dernière approche nécessite de connaître la composition en ingrédients des aliments concentrés, ce qui n'est pas le cas couramment observé dans la pratique, alors qu'il est toujours possible de déterminer la composition chimique d'un aliment.

Aucun effet significatif du niveau alimentaire, exprimé par la quantité ingérée en $\mathrm{g}$ M.S. $/ \mathrm{kg} \mathrm{P}^{0.75}$, sur la digestibilité de la matière organique n'a pu être mis en évidence malgré de larges variations de ce paramètre. Cette absence d'effets peut s'expliquer par le fait que les animaux recevaient, dans l'ensemble, des rations qui couvraient leurs besoins pour les différents éléments, et que la teneur en azote fermentescible des rations était élevée.

De plus, l'effet du niveau alimentaire sur la dM.O. est d'autant plus marqué que les aliments sont broyés finement et que les animaux ingèrent rapidement leur ration (Brown, 1966). Or, dans cette étude, les aliments sont à la disposition des animaux en permanence, le foin de luzerne est présenté sous forme longue et l'ensilage de maïs broyé grossièrement.

Ces résultats sont en accord avec ceux observés sur vaches laitières par BLoom et al. (1957 a et b), WiKToRsson (1971) et UDEN (1984) pour des animaux alimentés au prorata de leurs besoins, mais contredisent ceux de MOE, Reid \& TYRrel (1965), Brown (1966), Ekern (1972), Tyrrel \& Moe (1975), Lindgren (1981) et Colucci, Chase \& Van Soest (1982) qui font intervenir des animaux à l'entretien et en production dans leurs études du niveau alimentaire. Or, il semble que les animaux à faibles besoins comme les moutons et les vaches taries soient plus sensibles à une augmentation du niveau alimentaire que les animaux à niveau de production plus élevé comme 
les femelles laitières (Jordan \& Jenter, 1897 ; Leaver, Campling \& Holmes, 1969 ; Robertson \& Van Soest, 1975 ; Dulphy, 1983 ; Lassiter, HufFman \& Duncan, 1957). Cependant, cette absence d'effet sur la digestibilité de la matière organique serait à confirmer au niveau de la digestibilité de la fraction pariétale qui est plus sensible aux effets du niveau alimentaire d'après les études de Blaxter, Graham \& Wainman (1956), Robertson \& Van Soest (1975) ou Tyrrel \& Moe (1975).

\section{Conclusion}

Cette étude présente l'intérêt de fournir un ensemble de modèles de prévision de la digestibilité des rations mixtes ingérées à des niveaux alimentaires très différents. Sept aspects importants semblent pouvoir être retenus pour les utilisateurs :

- la lignine est le meilleur critère analytique de prédiction de la digestibilité de la matière organique quand les ingrédients des différents régimes étudiés sont de nature botanique très variée,

- les critères A.D.F. et cellulose brute ont des pouvoirs de prédiction de dM.O. analogue,

- le schéma de Van Soest permet de mieux cerner les facteurs de variation de la dM.O. que le schéma de Weende et d'obtenir une prévision aussi bonne, si ce n'est meilleure, que dans le cas de fourrages étudiés par famille végétale avec la méthode de Weende,

- la méthode de Van Soest permet un gain de précision de la valeur nutritive des rations mixtes de plus de $1 \mathrm{UFL} / 100 \mathrm{~kg}$ de matière sèche par rapport au schéma de Weende,

- la nature botanique des rations considérées et leur variabilité au sein d'une étude influe dans une large mesure sur les résultats obtenus et notamment sur la validité et le champ d'application des équations proposées,

- l'absence d'effet du niveau alimentaire testé globalement quand les animaux sont alimentés au prorata de leur niveau de production,

- la quasi absence d'effets liés aux interactions digestives.

D'un point de vue pratique, il importe de savoir si le gain de précision permis par la méthode de Van Soest justifie le coût analytique plus élevé qu'il entraîne.

Reçu en juin 1985.

Accepté en mars 1986.

\section{Remerciements}

Les auteurs tiennent à remercier M. J. Robelin, maître de recherches au laboratoire de la production de la viande au C.R.Z.V. de Theix, qui leur a communiqué le programme d'analyse de variance-covariance Seebeck et qui les a aidés dans l'interprétation des sorties de ce programme. 


\section{Summary \\ Predicting ability of different analytical criteria for digestibility \\ of mixed diets given to dairy goats}

The aim of this study was to determine the main factors of variation of organic matter digestibility of mixed diets given to dairy goats. It was based on experimental data collected by the laboratory of Nutrition and Feed from the «Institut National Agronomique Paris-Grignon 》 (INRA).

A total of 187 individual observations out of 224 were made in dairy goats and the remainder in dry goats. Forages used were lucerne hay and maize silage given either alone or together. This represented 31 diets differing in their cell-wall content. Therefore, the ranges of variation of cell-wall parameters (C.F., N.D.F., A.D.F. and A.D.L.) (table 1 and fig. 1, 2,3 ) as well as the percentages of cereals (including their by-products) and compound feeds were very large (table 2). Dry matter intake varied also considerably : 40 to $165 \mathrm{~g}$ D.M. $/ \mathrm{kg} \mathrm{W0.75}$. The different criteria of cell-wall analysis (crude fibre, N.D.F., A.D.F. and A.D.L.) were highly correlated (table 3). The highest correlation concerned C.F. and A.D.L. (0.97), and the lowest N.D.F. and A.D.L. (0.59).

Variations in the organic matter digestibility (table 4 and fig. $4,5,6$ ) were better explained by Van Soest's system than by Weende's. In fact, the different analytical parameters of the cell-wall evaluation had equivalent within-diet prediction potential, but the A.D.L. differed from the others in the between-diet correlation (table 5). Part of the residual variations, especially for Weende's system, can be explained by the botanical composition of the diet (fig. 7), as pointed out by the significant introduction of the percentage of cereals in the regression variables of the organic matter digestibility. The improvement of the prediction was less marked with Van Soest's system (fig. 8). This confirms the interest of the latter system to take into account better the differences of digestibility related to the botanical origin of the diets.

The feeding level had no statistically significant effect in this case where animals were fed according to their level of production (table 4, fig. 5). Using the proposed equations for mixed diets we obtained a precision of the same magnitude as the currently observed incertitude for experimental measurements of organic matter digestibility.

Key words : Digestibility, mixed diets, dairy ruminants, chemical analysis, prediction.

\section{Références bibliographiques}

Aerts J.V., De Brabander D.L., Cottyn B.G., Buysse F.X., 1977. Comparison of laboratory methods for predicting the organic matter digestibility of forages. Anim. Fecd Sci. Technol., 2, 337-350.

AFNOR, 1981. Cellulose brute. Méthode CEE. $4^{e}$ directive norme NF v 03-040. In : Aliments des animaux. Méthodes d'analyse. Ed. AFNOR.

Axelsson J., 1951. Effect of level of nutrition on digestibility of foodstuffs in different trials. Kungl. Lantbrukshoegsk. Ann., 18, 95-102.

Bailey R.W., UlyatT M.J., 1970. Pasture quality and ruminant nutrition. 2 Carbohydrates and lignin composition of detergent-extracted residues from pasture grasses and legumes. N.Z.J. Agric. Res., 13, 591-604.

Blaxter K.L., Graham N. Mc C., Wainman F.W., 1956. Some observations on the digestibility of food by sheep, and on related problems. Br. J. Nutr., 10, 69-91. 
Bloom S., Jacobson N.L., Allen R.S., McGilliard L.D., Homeyer P.G., 1957 a. Effects of various hay : concentrate ratios on nutrient utilization and production responses of dairy cows. I. Relationships among feeding level, predicted producing ability, and milk production. J. Dairy Sci., 40, 81-94.

Bloom S., Jacobson N.L., Allen R.S., McGilliard L.D., Homeyer P.G., 1957 b. Effects of various hay : concentrate ratios on nutrient utilization and production responses of dairy cows. II. Observations on ration digestibility and on the excretion pattern of chromic oxide. J. Dairy Sci., 40, 240-251.

Brown L.D., 1966. Influence of intake on fecd utilization. J. Dairy Sci., 49, 223-230.

CHARLET-LeRY G., 1969. Methods for determination of digestibility coefficients of feeds for ruminants. EAAP Report $\mathrm{n}^{\circ} 1$ from the study commission on animal nutrition, 1969, $33 \mathrm{pp}$.

Colucci P.E., Chase L.E., Van Soest P.J., 1982. Feed intake, apparent digestibility and rate of particulate passage in dairy cattle. J. Dairy Sci., 65, 1445-1456.

Dimarouilly C., Andr!eu J., Sauvant D., 1978. Composition et valeur nutritive des aliments. In : Alimentation des ruminants. Ed. INRA Publications, route de Saint-Cyr, 78000 Versailles, pp. 469-518.

DULPhy J.P., 1983. Les interactions digestives induites par les aliments concentrés : observations sur ovins. In : Quels aliments concentrés pour les vaches fortes productrices de lait? CAAA, 13 octobre 1983, ed. by ADEPRINA, 16, rue Claude-Bernard, 75231 Paris Cedex 05.

EDWARDS C.S., 1973. Determination of lignin and cellulose in forages by extraction with triethylène glycol. J. Sci. Food Agric., 24, 381-388.

EKERN A., 1972. Feeding of high yielding dairy cows. IV. Ration digestibility in high yielding cows fed at different levels of intake. Meld. orges Landbrukshogsk., 150, 1-18.

Giger S., Sauvant D., Dorleans M., Morand-Fehr P., 1979. Détermination semi-automatique des constituants membranaires des aliments concentrés par la méthode de Van Soest. 30th meeting of EAAP, Harrogate 23-26, July 1979.

Giger S., 1985. Les méthodes de dosage de la lignine utilisées en alimentation animale. Ann. Zootech., 34, 85-122.

Giger S., Sauvant D., Hervieu J., Dorleans M., 1986. Valeur alimentaire du foin de luzerne pour la chèvre. Bull. Theix (sous presse).

Gordon A.J., NeudoerfFer T.S., 1973. Chemical and in vivo evaluation of a brown midrib mutant of Zea mais. I. Fibre, lignin and aminoacid composition and digestibility for sheep. J. Sci. Food Agric., 24, 565-577.

Gordon A.J., 1975. A comparison of some chemical and physical properties of alkali lignins from grass and lucerne hays before and after digestion by sheep. J. Sci. Food Agric., 26, 1551-1559.

INRA, 1978. Tableaux de la valeur nutritive des aliments. In : Alimentation des ruminants. Ed. INRA Publications, route de Saint-Cyr, 78000 Versailles, pp. 519-555.

INRA, 1981. Tables de prévision de la valeur alimentaire des fourrages. In : Prévision de la valeur nutritive des aliments des ruminants. Ed. INRA Publications, route de SaintCyr, 78000 Versailles, pp. 363-549.

JARRIGE R., 1981. Les constituants glucidiques des fourrages : variations, digestibilité et dosage. In : Prévision de la valeur nutritive des aliments des ruminants. Ed. INRA Publications, route de Saint-Cyr, 78000 Versailles, pp. 13-40.

Jordan W.H., Jenter C.G., 1897. Digestion and feeding experiments. New York Agr. Exp. Eta. Bull., 141, cité par Schneider et Flatt, 1975.

Kim J.T., Gillingham J.T., Loadholt C.B., 1967. Difference in composition between crude fibre and acid detergent fibre. J. Assoc. Off. Anal. Chem., 50, 340-343.

KirChgessNer R.M., Kellner R.J., 1977. Zur Schaetzung der umsetzbaren Energie von Gruen und Raufutter mit einfachen Kenndaten. Z. Tierphysiol. Tierernaehrung Futtermittelk, 38, 297-301.

Lassiter C.A., Huffman C.F., Duncan C.W., 1957. The effect of varying hay-grain ratios and levels of feed intake in feed utilization of dairy cows. J. Dairy Sci., 40, 611 (Abstr.). 
LAUbE W., 1960. Neue Untersuchungen ueber den Einfluss der pflanzlichen Geruestsubstanzen auf die Verdaulichkeit von Gruenfutterstoffen. Arch. Tierernaehr., 10, 99-112.

Leaver J.D., Campling R.C., Holmes W., 1969. The effect of level of feeding on the digestibility of diets for sheep and cattle. Anim. Prod., 11, 11-18.

Lebart L., Fenelon J.P., 1971. Statistique et informatique appliquées. Ed. Dunod, 440 pp.

Lindgren E., 1981. Prediction of energy value of mixed diets for lactating cows from digestibility experiments with sheep. Swed. J. Agric. Res., 11, 177-184.

Malossini F., Martilotti F., Ferrari P., Rauzi G.M., 1973. Stima della digeribilita e del valore nutritivo di fieni prolifiti con diversi metodi ed in particolare con il metodo di Van Soest. Ann. Ist. Sper. Zootec., 6, 21-49.

Malossini F., Bartocci S., Verna M., Mazziotti di Celso P., 1979. Relazione fra contenuto in fibra grezza e digeribilita in vivo della sostanze organica. Ann. Ist. Sper. Zootec., 12, 155-175.

Minson D.J., 1982. Effect of chemical composition on feed digestibility and metabolizable energy. Nutr. Abstr. Rev. (B), 52, 591-615.

Moe P.W., Reid J.T., Tyrrell H.F., 1965. Effect of level of intake on digestibility of dietary energy by high-producing cows. J. Dairy Sci., 48, 1053-1061.

Nehring K., HofFManN B., 1969. Untersuchungen zur Weiterentwicklung der Futtermittelanalyse. I. Die Problematik der Futtermitteluntersuchung. Arch. Tierernaehr., 19, $561-570$.

Nehring K., 1974. Ueber den Futterwert verschiedener Getreideprodukte. V. Die Errechnung des Gehaltes an Netto-Energie-Fett (NEF) aus einfachen Kenndaten bei den verschiedenen Getreideprodukten. Arch. Tierernaehr., 24, 429-441.

Nehring K., 1975. Die Ermittlung des Futterwertes auf Grundeinfacher Kenndaten. I. Die Ermittlung der Verdaulichkeit bei Gruenfutterstoffen und ihren Konservaten. Arch. Tierernaehr., 25, 207-229.

Nordfeldt S., Svanberg O., Claesson O., 1949. Studies regarding the analysis of crude fibre. Acta Agric. Swed., 3, 137-177.

Paquay R., de Baere R., Lousse A., 1966. Recherches statistiques sur la digestibilité des éléments de la ration. Ann. Méd. Vét., 110, 13-55.

Porter P., Singleton A.G., 1971. The degradation of lignin and quantitative aspects of ruminant digestion. Br. J. Nutr., 25, 3-14.

PROTECTOR, 1980. Tables de composition des matières premières destinées à l'alimentation animale. 1. Valeurs chimiques. Aliments Protector S.A., Bruxelles, 84 p.

R.R.I., Rowett Research InStitute, 1975. Feedingstuffs evaluation unit. First report 1975, ed. DAFS, Edimburgh, 58 pp.

R.R.I., RowetT RESEARCH INSTITUTE, 1978. Feedingstuffs evaluation unit. Second report 1978, ed. DAFS, Edimburgh, $36 \mathrm{pp}$.

Robertson J.B., Van Soest P.J., 1975. A note on digestibility in sheep as influenced by level of intake. Anim. Prod., 21, 89-92.

SchneIder B.H., Flatt W.P., 1975. Factors affecting digestibility. In : The evaluation of feeds through digestibility experiments. Ed. The University of Georgic Pros, Athens, 237-267.

SAUVANT D., 1980. Rapport sur la valeur énergétique et azotée des aliments concentrés. $30^{\circ}$ Congrès FEZ, Commission nutrition, Munich 1980.

Sauvant D., 1981. Prévision de la valeur énergétique des aliments concentrés et composés pour les ruminants. In : Prévision de la valeur nutritive des aliments des ruminants. Ed. INRA Publications, route de Saint-Cyr, 78000 Versailles, pp. 237-258.

Theander O., Aman P., 1980. Chemical composition of some forages and various residues from feeding value determinations. J. Sci. Food Agric., 31, 31-37.

Tyrrell F.F., Moe P.W., 1975. Effect of intake on digestive efficiency. J. Dairy Sci., 58, 1151-1163. 
UDEN P., 1984. Digestibility and digesta retention in dairy cows receiving hay or silage at varying concentrate levels. Anim. Feed Sci. Technol., 11, 279-291.

VAN SOEST P.J., 1963. Use of detergents in the analysis of fibrous feeds. II. A rapid method for the determination of fibre and lignin. J. Assoc. Off. Anal. Chem., 40, 829-835.

VAN SoEST P.J., WINE R.H., 1967. Use of detergents in the analysis of fibrous feeds. IV. Determination of plant cell-wall constituents. J. Assoc. Off. Anal. Chem., 50, 50-55.

VAN SoEst P.J., 1978. Estimation of forages and feedstuffs in the laboratory. VI. International Veterinary Congress, Buenos Aires, Argentina, November 1978.

VenCl B., Flam F., 1981. Predicting the energy value of forages from digestibility in potassium hydroxide. Anim. Feed Sci. Technol., 6, 235-243.

Walker D.M., Hepburn W.R., 1955. The nutritive value of roughages for sheep. I. The relationship between the gross digestible energy and the chemical composition of hays. J. Agric. Sci., 45, 298-310.

Wiktorsson H., 1971. Digestibility experiments with dairy cows consuming different quantities of concentrates. J. Dairy Sci., 54, 374-378. 